\title{
Glaucoma-The Importance of Early Detection and Early Treatment
}

\author{
a report by \\ F Topouzis, MD and E Anastasopoulos, MD
}

Department of Ophthalmology, Aristotle University of Thessaloniki, Greece DOI: 10.17925/USOR.2007.02.00.12

\begin{abstract}
An Increasing Global Concern
Glaucoma is a major global health problem, and all types of the disease combined are the second leading cause of blindness worldwide, with approximately 6.7 million people being blinded as a result of the disease. ' With the predicted increase in life expectancy, the anticipated number of people becoming blind from the disease will rise substantially in the near future. Despite increasing public health awareness and the availability of advancedtechnology diagnostic tests in developed countries, a high proportion of glaucoma cases remain undiagnosed in the community. Improved strategies for achieving earlier and more accurate diagnosis of glaucoma will facilitate the prompt implementation of effective treatment options, and subsequently will minimize the anticipated rising burden of the disease in the near future.
\end{abstract}

The Problem of Early Detection of Glaucoma

Various population-based epidemiological studies have reported that more than $50 \%$ of glaucoma cases remain undiagnosed, even in developed countries. $^{2-5}$ High prevalence estimates of undiagnosed glaucoma are consistent with the lack of cost-effective screening methods for glaucoma. Despite being a major risk factor for glaucoma, elevated intraocular pressure (IOP) lacks sensitivity and specificity in glaucoma diagnosis since it is not present in a high proportion of affected patients at the time of diagnosis, while many people without glaucoma present with elevated IOP. Also, possible reasons for the high prevalence of undiagnosed glaucoma include the fact that glaucoma is a silent, asymptomatic disease.

Visual function may not deteriorate significantly until advanced stages of the disease. However, even in the advanced stages of the disease glaucoma can remain undiagnosed due to lack of regular visits to an ophthalmologist or lack of thoroughness at the eye examination. In a recent population-based study of the major eye diseases in Greece-the Thessaloniki Eye Study — a high prevalence of undiagnosed glaucoma (approximately 50\%) was reported. The majority of cases were not at early glaucoma stage: mean cup-to-disc $(\mathrm{c} / \mathrm{d})$ ratio of undiagnosed glaucoma cases was 0.7 , and mean visual field Advanced Glaucoma Intervention Study (AGIS) score was 6.4.

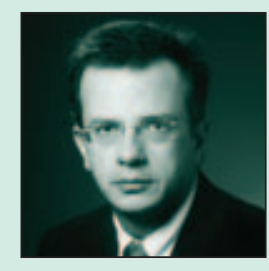

Fotis Topouzis, MD, is Assistant Professor of Ophthalmology at the Aristotle University of Thessaloniki, Greece. With interests in glaucoma and age-related macular degeneration, he is Founder and Head of the Laboratory of Research and Clinical Applications in Ophthalmology and the principal investigator for two large population-based studies: the Thessaloniki Eye Study and the Eureye Study.

E: ftopouzis@otenet.g
A subsequent analysis of factors associated with non-diagnosis showed that glaucoma patients who had not seen an eye doctor during the previous year had six-fold increased odds of being undiagnosed compared with patients who had visited an eye doctor during the last year. ${ }^{6}$ Also, primary open-angle glaucoma (POAG) patients had a fourfold increased risk of being undiagnosed compared with pseudoexfoliative glaucoma patients, indicating that the thoroughness at the eye examination and the performance of the eye doctors in diagnosing glaucoma may increase when pseudo-exfoliative material is present. Furthermore, the Visual Impairment Project (VIP), a population-based study in Australia, reported that an increased passage of time since the last visit to an eye-care provider was associated with an elevated risk of undiagnosed glaucoma.? Additionally, the type of eye professional seen within the previous 12 months was statistically significantly different between the diagnosed and undiagnosed glaucoma groups, with the undiagnosed group being more likely to be examined by an optometrist. ${ }^{8}$ Increased patient motivation for regular visits to the eye-care professionals, improved training of the latter, and standardization of examination protocols could facilitate better detection rates with conventional clinical examination diagnostic tools.

\section{The Problem of Detection of Early Glaucoma}

The structural changes at the optic nerve head and nerve fiber layer loss are the most common first signs of glaucoma neuropathy and usually precede the functional visual field deterioration. Subjective estimation of the $\mathrm{c} / \mathrm{d}$ area ratio by biomicroscopy has long been used to quantify the degree of cupping in glaucoma in the clinical setting. However, this method remains essentially qualitative, and detection of early glaucomatous signs and progressive glaucomatous changes in the optic disc using this method is extremely difficult due to poor sensitivity and high inter- and intra-observer variability.

Photography of the optic disc is a traditional method of documenting the appearance of and demonstrating longitudinal changes in the disc. However, these non-quantitative methods require subjective clinical interpretation and sometimes lack the sensitivity needed to detect the subtle structural changes that occur in early glaucoma stages. In the Thessaloniki Eye Study, a smaller c/d ratio was strongly associated with an increased risk of undiagnosed POAG, which highlights the inherited difficulties of identifying the early signs of the disease in subjects with small cupping.

Over the past 15 years, more objective ocular imaging techniques have been developed in an effort to provide accurate, reproducible quantitative measurements of the optic disc and the retina nerve fiber 
layer structure. The major imaging technologies-scanning laser polarimetry (SLP), confocal laser scanning ophthalmoscopy (CSLO), and optical coherence tomography (OCT) — provide sensitive measurements of these structural features. Even though the diagnostic accuracy of these devices is high — comparable to that of glaucoma experts9,10 —and can detect glaucoma-related structural changes before the development of visual field changes, ${ }^{11}$ their wide use as screening tools for glaucoma diagnosis in the community is not considered cost-effective at this time.

\section{The Importance of Early Glaucoma Treatment}

Millions of people remain exposed to an increased risk of blindness or significant visual impairment and reduced quality of life if the condition is undiagnosed and left untreated. In a study of the natural history of glaucoma in the West Indies, $16 \%$ of glaucoma patients or suspects progressed to blindness in at least one eye and $5 \%$ to bilateral blindness over a 10 -year period. ${ }^{12}$ Over $50 \%$ of eyes that progressed to end-stage glaucoma had no or minimal visual field loss at baseline.

Once ocular hypertension or glaucoma is diagnosed, the aim of therapy is to prevent disease progression. European Glaucoma Society (EGS) guidelines recommend that the goal of treatment should be to provide lifetime preservation of visual function and quality of life at a sustainable cost. ${ }^{13}$ Large randomized clinical trials have proved that IOP reduction is effective in decreasing risk of disease progression.

In the Ocular Hypertension Treatment Study (OHTS), a 20\% decrease of IOP from baseline in subjects with ocular hypertension reduced the risk of progression to glaucoma by 50\%. ${ }^{14}$ In the Early Manifest Glaucoma Trial (EMGT), early treatment and a $25 \%$ decrease in IOP in patients with early and newly diagnosed glaucoma reduced and delayed glaucoma progression over a six-year period from $62 \%$ in the untreated group to $45 \%$ in the treated group..$^{15}$

The use of topical medications to lower IOP is the first-line strategy used to achieve this goal. A target IOP must be defined for each patient based on the reduction of IOP considered adequate to prevent further disease progression. According to EGS guidelines, the target IOP should be determined with reference to factors such as the age and the life expectancy of the patient, the IOP at baseline before treatment, the stage of the optic nerve damage, and the rate of disease progression. ${ }^{13}$
Nevertheless, predicting the rate of disease progression is a major challenge in the management of ocular hypertension and glaucoma. In the OHTS, almost $90 \%$ of the untreated subjects with ocular hypertension did not progress to glaucoma, while $4.4 \%$ of the treated progressed despite treatment. ${ }^{14}$ Following onset of the disease, the risk and rate of further disease progression also vary considerably between individuals. The EMGT found over a six-year period that $38 \%$ of patients without treatment did not progress, while $45 \%$ of those with treatment progressed..$^{15}$

As progression is difficult to predict on an individual basis, treatment decisions are usually guided by a global assessment of various risk factors. However, one of the most important compromises in glaucoma management is that changes in treatment decisions are usually made after signs of progression are identified. By treating patients as early as possible and making the appropriate treatment choice, protection against further disease progression is maximized. Decisions on initial medical therapy should consider not only the IOP-lowering efficacy but also the long-term ocular tolerability and compliance of the patient.

Prostaglandin derivates are the most commonly used first-choice medical therapy for glaucoma, mainly due to the superior IOP-lowering efficacy, the lower incidence of systemic side effects, and better compliance-due to the one dose per day regime-compared with the other glaucoma drugs.

Early detection of glaucoma is essential not only to maximize the benefits of treatment for patients, but also to minimize the economic burden of glaucoma in society. Recent data have shown that the costs of treating glaucoma in Europe and the US rise as the severity of disease increases, and the annual cost of glaucoma increases relative to how advanced the disease is at the time of diagnosis. ${ }^{16,17}$ Thus, significant potential savings and reductions in annual healthcare burdens are possible if patients are diagnosed and treated at earlier stages of glaucoma.

Since a high proportion of glaucoma cases are undiagnosed in the community, early glaucoma detection remains a challenge. Strategies to improve screening for glaucoma and glaucoma detection in everyday practice are necessary in order to ensure that the potential benefits arising from early treatment will be implemented.
1. Quigley HA, Number of people with glaucoma worldwide, Br J Ophthalmol, 1996;80(5):389-93.

2. Tielsch JM, Sommer A, Katz J, et al., Racial variations in the prevalence of primary open-angle glaucoma. The Baltimore Eye Survey, JAMA, 1991;266:369-74.

3. Mitchell P, Smith W, Attebo K, Healey PR, Prevalence of openangle glaucoma in Australia. The Blue Mountains Eye Study, Ophthalmology, 1996;103:1661-9.

4. Dielemans I, Vingerling JR, Wolfs RCW, et al., The prevalence of primary open-angle glaucoma in a population-based study in the Netherlands. The Rotterdam Study, Ophthalmology, 1994;101:1851-5.

5. Topouzis F, Wilson MR, Harris A, et al., Prevalence of open angle glaucoma in Greece. The Thessaloniki Eye Study, Am J Ophthalmol, 2007;144:511-19.

6. Topouzis F, Harris A, Coleman AL, et al., Factors associated with undiagnosed open angle glaucoma. The Thessaloniki Eye Study, Am J Ophthalmol, in press.

7. Wong EYH, Keeffe JE, Rait JL, et al., Detection of undiagnosed glaucoma by eye health professionals, Ophthalmology, 2004;111:1508-14.

8. Weih LM, Nanjan M, McCarty C, Taylor HR, Prevalence and predictors of open-angle glaucoma. Results from the visual impairment project, Ophthalmology, 2001;108:1966-72.

9. Medeiros FA, Zangwill LM, Bowd C, Weinreb RN, Comparison of the GDx VCC scanning laser polarimeter, HRT II confocal scanning laser ophthalmoscope, and stratus OCT optical coherence tomograph for the detection of glaucoma, Arch Ophthalmol, 2004;122(6):827-37.

10. Badala F, Nouri-Mahdavi $K$, Raoof DA, et al., Optic disk and nerve fiber layer imaging to detect glaucoma, Am J Ophthalmol, 2007; in press.

11. Bowd C, Zangwill LM, Medeiros FA, et al., Confocal scanning lase ophthalmoscopy classifiers and stereophotograph evaluation for prediction of visual field abnormalities in glaucoma-suspect eyes, Invest Ophthalmol Vis Sci, 2004;45(7): 2255-62.

12. Wilson MR, Kosoko O, Cowan CL Jr., et al., Progression of visual field loss in untreated glaucoma patients and glaucoma suspects in St. Lucia, West Indies, Am J Ophthalmol, 2002;134(3):399-405.

13. European Glaucoma Society Terminology and Guidelines for Glaucoma, second edition, Savona, Italy: European Glaucoma Society, 2003.

14. Gordon MO, Beiser JA, Brandt JD, et al., The Ocular Hypertension Treatment Study: baseline factors that predict the onset of primary open-angle glaucoma, Arch Ophthalmol, 2002;120(6):714-20.

15. Heijl A, Leske MC, Bengtsson $B$, et al., Reduction of intraocular pressure and glaucoma progression: results from the Early Manifest Glaucoma Trial, Arch Ophthalmol, 2002;120(10): 1268-79.

16. Lee PP, Kelly SP, Mills RP, et al., Glaucoma in the United States and Europe: predicting costs and surgical rates based upon stage of disease, J Glaucoma, 2007;16(5):471-8.

17. Traverso CE, Walt JG, Kelly SP, et al., Direct costs of glaucoma and severity of the disease: a multinational long term study of resource utilisation in Europe, Br J Ophthalmol, 2005;89(10): $1245-9$. 